\title{
The Processing of Hexagonally Sampled Signals with Standard Rectangular Techniques: Application to 2-D Large Aperture Synthesis Interferometric Radiometers
}

Adriano Camps, Student Member, IEEE, Javier Bará, Ignasi Corbella Sanahuja, Member, IEEE, and Francesc Torres

\begin{abstract}
In Earth observation programs there is a need of passive low frequency ( $L$-band) measurements to monitor soil moisture and ocean salinity with high spatial resolution 10-20 $\mathrm{Km}$, a radiometric resolution of $1 \mathrm{~K}$ and a revisit time of 1-3 days [1]. Compared to total power radiometers aperture synthesis interferometric radiometers are technologically attractive because of their reduced mass and hardware requirements. In this field it should be mentioned the one-dimensional (1-D) linear interferometer ESTAR developed by NASA [2] and MIRAS a two-dimensional (2-D) Y-shaped interferometer currently under study by European Space Agency (ESA) [3]. Interferometer radiometers measure the correlation between pairs of nondirective antennas. Each complex correlation is a sample of the "visibility" function which, in the ideal case, is the spatial Fourier transform of the brightness temperature distribution. Since most receiver phase and amplitude errors can be hardware calibrated, Fourier based iterative inversion methods will be useful when antenna errors are small, their radiation voltage patterns are not too different, and mutual coupling is small. In order to minimize on-board hardware requirements-antennas, receivers and correlators-the choice of the interferometer array shape is of great importance since it determines the $(u, v)$ sampling strategy and the minimum number of visibility samples required for a determined aliasing level. In this sense, Y-shaped and triangularshaped arrays with equally spaced antennas are optimal. The main contribution of this paper is a technique that allows us to process the visibility samples over the hexagonal sampling grids given by Y-shaped and triangular-shaped arrays with standard rectangular FFT routines. Since no interpolation processes are involved, the risk of induced artifacts in the recovered brightness temperature over the wide field of view required in Earth observation missions is minimized and signal to noise ratio (SNR) is preserved.
\end{abstract}

Index Terms - Hexagonal Fourier transform, interferometry, reciprocal basis, visibilities.

\section{BASIC EQUATIONS}

$\mathbf{T}$ HE RELATIONSHIP between a visibility sample, the signals involved in the measurement, and the brightness temperature distribution for an ideal interferometer is given

Manuscript received August 8, 1995; revised May 28, 1996. This work was supported by the European Space Agency ESA within the framework of ESA MIRAS Rider 2 activities with MATRA MARCONI SPACE as main contractor.

The authors are with the Universitat Politècnica de Catalunya, Department of Signal Theory and Communications, 08034 Barcelona, Spain.

Publisher Item Identifier S 0196-2892(97)00364-1.

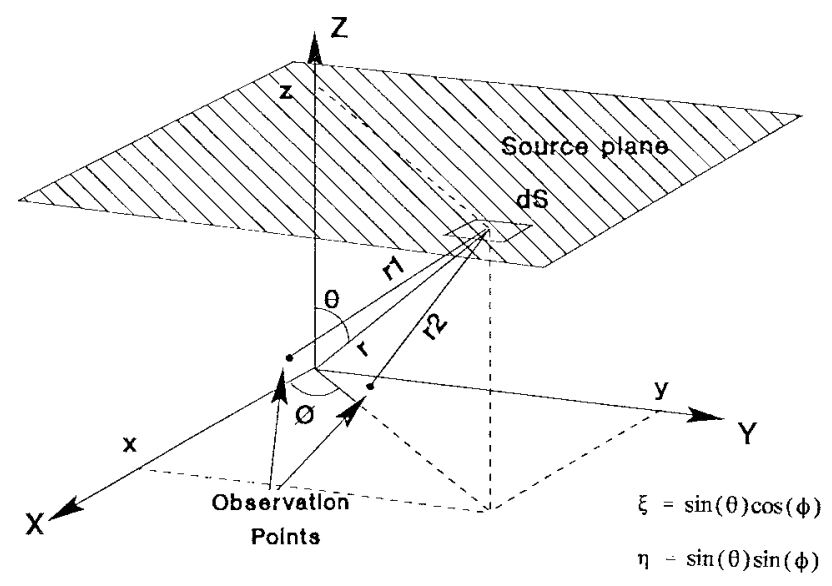

Fig. 1. Geometry of the interferometer radiometer and related terms.

by [2], [4]

$\begin{aligned} V(u, v) & =\frac{1}{2} E\left[S_{a_{1}}(t) S_{a_{2}}^{*}(t)\right] \\ & =\iint_{\xi^{2}+\eta^{2} \leq 1} T(\xi, \eta) \tilde{r}\left(-\frac{u \xi+v \eta}{f_{o}}\right) e^{-j 2 \pi(u \xi+v \eta)} \cdot d \xi d \eta\end{aligned}$

(1)

$T(\xi, \eta)=\frac{T_{B}(\xi, \eta)}{\sqrt{1-\xi^{2}-\eta^{2}}}\left|F_{n}(\xi, \eta)\right|^{2}$

where $S_{a 1}$ and $S_{a 2}$ are the analytic signals of the voltages collected by antennas 1 and $2,(u, v)=\left(X_{2}-X_{1}, Y_{2}-\right.$ $\left.Y_{1}\right) / \lambda$ is the spacing between the two antennas in wavelengths, $(\xi, \eta)=(\sin \theta \cos \phi, \sin \theta \sin \phi)$ are the directing cosines with respect to $(X, Y)$ axes (Fig. 1$), T_{B}(\xi, \eta)$ is the brightness temperature, $T(\xi, \eta)$ is the modified brightness temperature, $1 / \sqrt{1-\xi^{2}-\eta^{2}}$ is the obliquity factor $F_{n}(\xi, \eta)$ is the normalized antenna voltage pattern, $\widetilde{r}(t)=r(t) e^{-j 2 \pi f_{o} t}$ is the fringe-wash function that takes into account spatial decorrelation effects and depends on receiver's responses through

$$
r(t)=\int_{0}^{\infty}\left|H_{n}(f)\right|^{2} e^{j 2 \pi f t} d f
$$

and $H_{n}(f)$ is the receiver's normalized band-pass voltage transfer function $\left(\left|H_{n}(f)\right|_{\max }=1\right)$. Note that the integral 
Y-shaped array

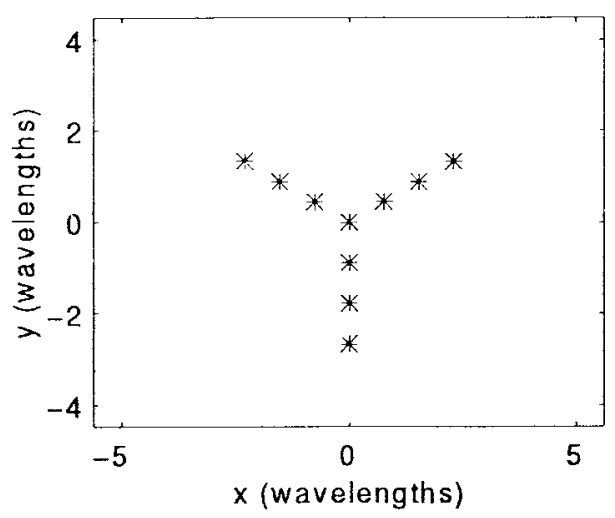

(a) triangular-shaped array

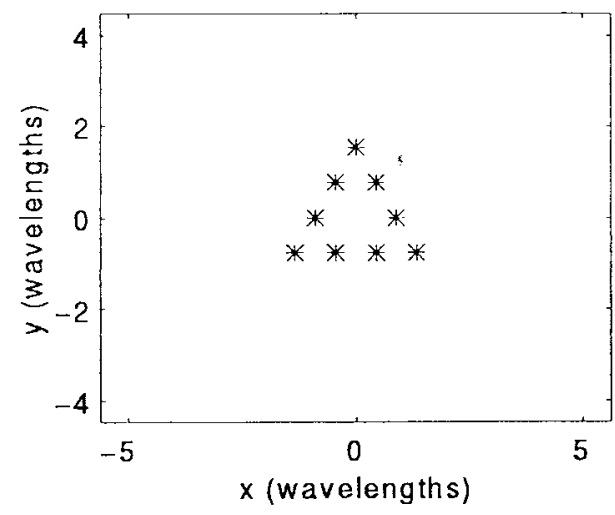

(b)

Fig. 2. Configuration of (a) Y-shaped and (b) triangular-shaped arrays with three antennas per arm spaced $0.89 \lambda$.

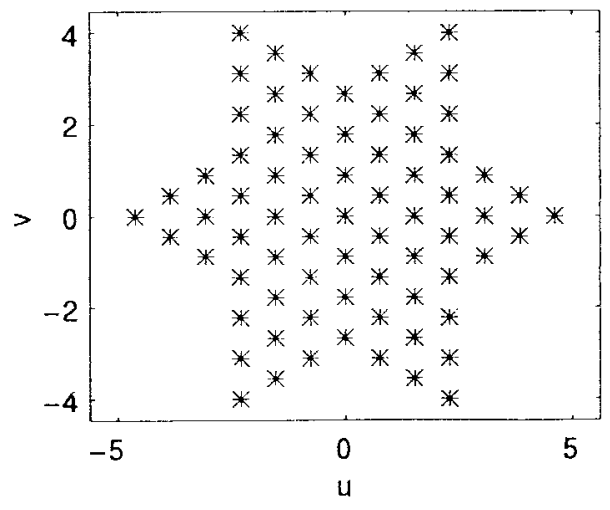

(a)

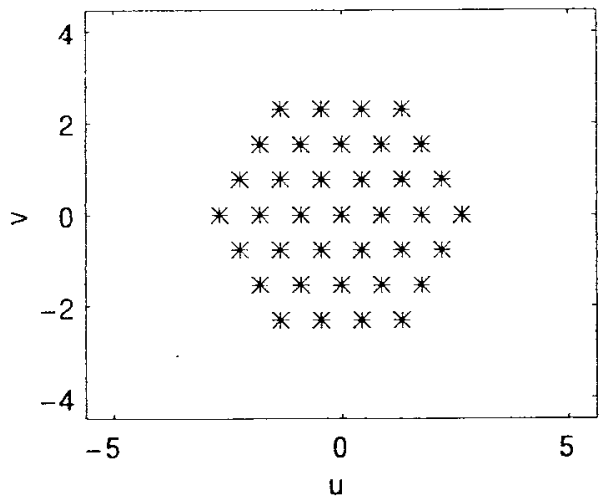

(b)

Fig. 3. Spatial frequency coverage for the arrays shown in Fig. 2.

limits in (3) range from zero to infinite since the analysis is done with analytic signals. When decorrelation effects are negligible $(\tilde{r}(t) \approx 1)$ and all the antennas have the same voltage radiation pattern (1) becomes a Fourier transform between the visibility function and the modified brightness temperature $T(\xi, \eta)$ :

$$
T(\xi, \eta)=F^{-1}[V(u, v)] .
$$

The whole space maps into the unit the circle in the $(\xi, \eta)$ plane, consequently any modified brightness temperature distribution $T(\xi, \eta)$ will be supported by

$$
\begin{aligned}
\xi^{2}+\eta^{2} & =\sin ^{2}(\theta) \cos ^{2}(\phi)+\sin ^{2}(\theta) \sin ^{2}(\phi) \\
& =\sin ^{2}(\theta) \leq 1 .
\end{aligned}
$$

signal theory it is known that this class of signals are optimally sampled by using an hexagonal grid, in the sense that this grid requires the minimum density of $(u, v)$ samples to recover it with a specified aliasing level $(13.4 \%$ less samples than rectangular sampling) [5], [6]. Y-shaped and triangular-shaped arrays [Fig. 2(a) and (b)] produce visibility samples over a hexagonal grid in the spatial frequencies domain $(u, v)$ [Fig. 3(a) and (b)]. Fig. 3(a) shows the $(u, v)$ coverage in the case of MIRAS breadboard, a Y-shaped interferometer radiometer with three antennas per arm spaced 0.89 wavelengths [Fig. 2(a)]. As it can be seen in Fig. 3(a) and (b), for the same hardware complexity, similar number of antennas and receivers, the spatial resolution obtained for a Y-shaped array is better than that for a triangular-shaped array, since the spatial frequency coverage is larger in the first case. On the other hand, triangular-shaped arrays cover a complete hexagonal period, while $\mathrm{Y}$-shaped arrays have missing $(u, v)$ samples between the star points [Fig. 3(a)]. These missing values should be extrapolated in some way [7] in order to prevent the artifacts induced by the $(u, v)$ star-shaped lowpass window. However, this is an important effect only in small arrays where the star-shaped window effectively lowpass filters the visibility function. For large arrays, such as the planned MIRAS space borne instrument, with 43 antennas per arm, less than $0.7 \%$ visibility power is not collected by the array and this effect is negligible [7]. 
From now on we will focus only on Y-shaped arrays, as MIRAS, where the visibility function is sampled over the grid

$$
\begin{aligned}
u & =\frac{\sqrt{3}}{2} d k_{1} ; \quad k_{1}, k_{2}: 0 \cdots N_{T} \\
v & =\frac{d}{2}\left(-k_{1}+2 k_{2}\right) \\
V(u, v) & \triangleq V(\bar{k}) ; \quad \bar{k}=\left(k_{1}, k_{2}\right)
\end{aligned}
$$

where $N_{T}=3 N_{E L}+1$ is the total number of antennas, $N_{E L}$ is the number of antennas in each arm of the $Y$ array and $d$ is the spacing in wavelengths between adjacent antennas. It should be pointed out that since the brightness temperature distribution is obtained by an inverse Fourier transform, it can suffer from aliasing, which is determined by the spacing between adjacent antennas " $d$." This effect will be studied in detail in Section II. In the next section we will also show how standard rectangular FFT routines can be applied to the hexagonal $(u, v)$ coverage given by Y-shaped arrays, avoiding the need of interpolations, preserving signal to noise ratio and retaining the benefits of the hexagonal sampling grid.

\section{HeXagonal FFT, Smith-Normal DECOMPOSITION AND RECIPROCAL BASIS}

Let's first recall some concepts about 1-D Fourier transforms. The DFT of a bandlimited 1-D sequence $x(n)$ of length $N$ obtained by sampling the signal $x(t)$ each $T s$ seconds, gives $N$ samples of the spectrum of the signal $\widetilde{x}(t)$, the periodic extension of $x(t)$ in time interval $[0, N \cdot T s]$. These $N$ frequency samples are a single period of the periodic spectrum. As they come out the FFT these samples are swapped: the samples corresponding to negative frequencies appear right after the positive ones. By padding the sequence $x(n)$ with zeros a smoother spectrum's shape can be obtained without adding new information. In bandlimited 2-D sequences, in addition to the number of zero padded samples that can be put, the periodic extension of the spectrum itself can be chosen (Figs. 4 and 5). This means that the known spectrum samples do not need to be repeated periodically along the " $u$ " and " $v$ " axes. The way a spectrum is repeated is characterized by its periodicity matrix $N$ :

$$
\tilde{V}(\bar{k})=\tilde{V}(\bar{k}+\bar{N} \cdot \bar{r})
$$

where $\tilde{V}(\bar{k})$ is the periodic extension of $V(k), N$ is a nonsingular integer matrix called the periodicity matrix and $\boldsymbol{r}$ is an integer vector.

The number of samples, zero or not, in one period is given by $\operatorname{det}(\boldsymbol{N})$, for a given periodicity matrix $N$ [6]. For an $Y$ array, the number of nonredundant visibility samples is given by

$$
N_{V}=6 N_{E L}^{2}+6 N_{E L}+1
$$

and the number of missing samples to be initially padded with zeros is

$$
N_{V-}=\operatorname{det}(\overline{\bar{N}})-N_{V}
$$

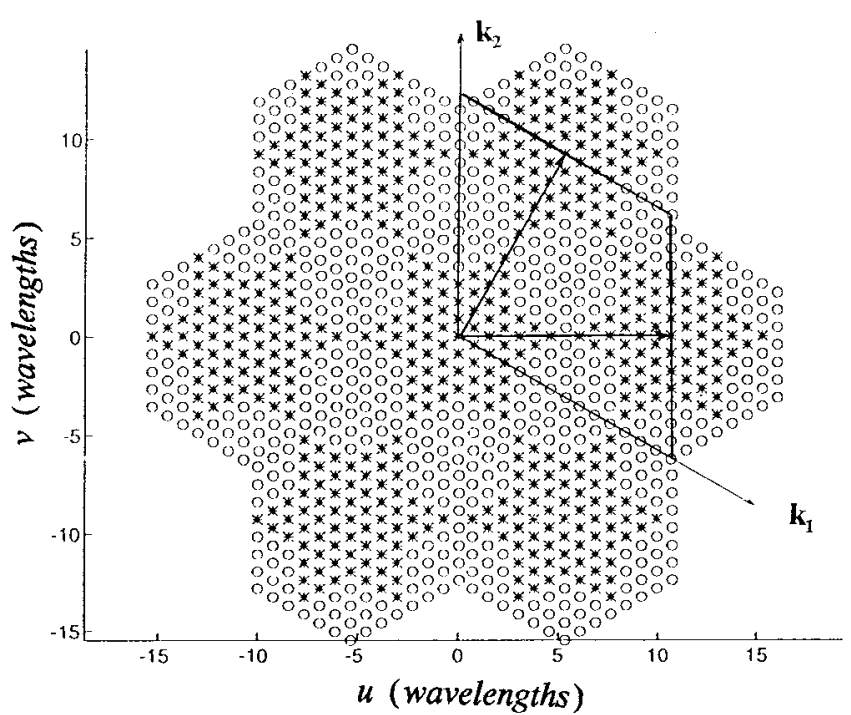

Fig. 4. Periodic extension of the $(u, v)$ coverage given in Fig. 3(a). HFFT required.

which should be minimized by properly choosing the periodicity matrix $N$. The choice of $N$ is not unique. One possible choice is presented in Fig. 4 for $N_{E L}=3$. For this periodic extension, $N$ is given by

$$
\overline{\bar{N}}=\left[\begin{array}{cc}
N_{T} & 2 N_{T} \\
2 N_{T} & N_{T}
\end{array}\right] ; \quad \operatorname{det}(\overline{\bar{N}})=3 N_{T}^{2}
$$

which leads to Mersereau's hexagonal FFT algorithm [5], [6].

A more general approach can be used with the help of the Smith Normal decomposition [9], [10], which states that any nonsingular integer matrix $N$ can be diagonalized by pre- and post-multiplication by unimodular integer matrices $\boldsymbol{E}$ and $\boldsymbol{F}$ :

$$
\begin{gathered}
\overline{\bar{N}}=\overline{\bar{E}} \cdot \overline{\bar{\Lambda}} \cdot \overline{\bar{F}} \\
\operatorname{det}(\overline{\bar{E}})=\operatorname{det}(\overline{\bar{F}})=1 .
\end{gathered}
$$

$\Lambda$ is a diagonal matrix, thus any arbitrary fundamental period, not only that presented in Fig. 4, and over any arbitrary sampling grid, not only a hexagonal one, can be mapped into a rectangular one allowing rectangular FFT routines to be used in the reordered indexes $\boldsymbol{k}^{\prime}$ and $\boldsymbol{n}^{\prime}$ :

$$
\begin{aligned}
T(\bar{n}) & \triangleq T\left(\xi\left(n_{1}, n_{2}\right), \eta\left(n_{1}, n_{2}\right)\right) \\
& =\frac{1}{|\operatorname{det}(\overline{\bar{N}})|} \sum_{\bar{k}} \tilde{V}(\bar{k}) e^{j 2 \pi\left(\bar{k}^{T} \overline{\bar{N}}^{-1} \bar{n}\right)} \\
& =\frac{1}{|\operatorname{det}(\overline{\bar{N}})|} \sum_{\bar{k}} \tilde{V}(\bar{k}) e^{j 2 \pi\left(\left(\bar{k}^{T} \overline{\bar{F}}^{-1}\right) \overline{\bar{\Lambda}}^{-1}\left(\overline{\bar{E}}^{-1} \bar{n}\right)\right)} \\
& =\frac{1}{|\operatorname{det}(\overline{\bar{N}})|} \sum_{\bar{k}} \tilde{V}(\bar{k}) e^{j 2 \pi\left(\bar{k}^{\prime} \overline{\bar{N}}^{-1} \overline{n^{\prime}}\right)}
\end{aligned}
$$

where

$$
\overline{k^{\prime}} \triangleq\left(\bar{F}^{-1}\right)^{T} \bar{k}
$$

and:

$$
\overline{n^{\prime}} \triangleq \overline{\bar{E}}^{-1} \bar{n} .
$$




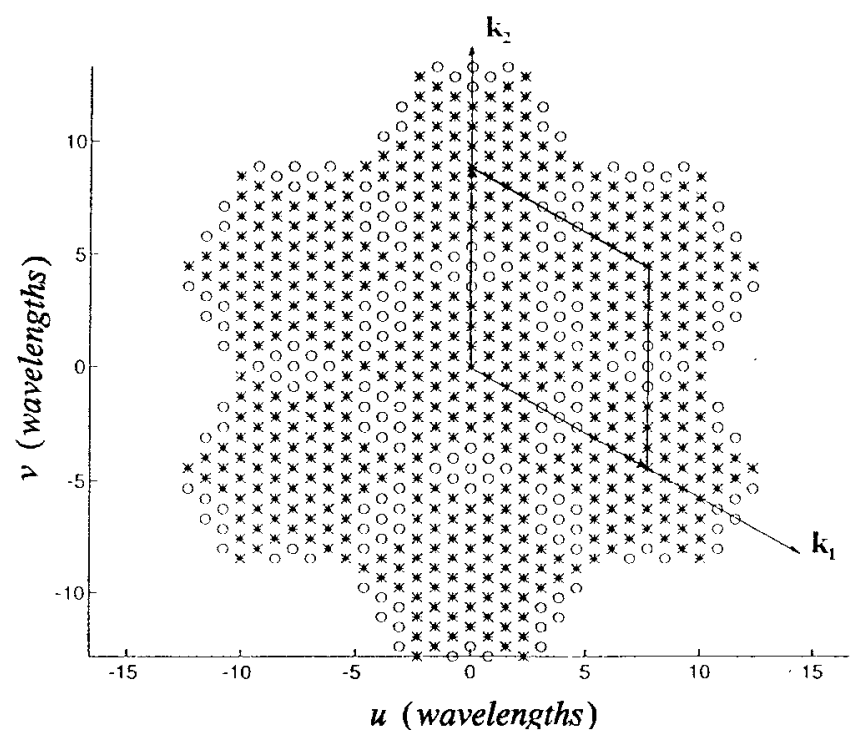

Fig. 5. Periodic extension of the $(u, v)$ coverage given in Fig. 3(a). Standard FFT.

The method proposed in this paper is based in the choice of an appropriate diagonal matrix $N$ that minimizes the number of samples in the periodic cell $\left(3 N_{T}^{2}\right)$ and the number of nonmeasured visibilities which must be initially padded with zeros $\left(3 N_{T}^{2}-N_{V}\right)$, allows to use standard rectangular FFT routines and avoids the indexes permutations required by the Smith Normal decomposition (14) and (15). Let's periodically extend the $(u, v)$ fundamental period as in Fig. 5. In this scheme measured visibility samples are repeated following the relation given below

$$
\begin{aligned}
\tilde{V}\left(u^{\prime}, v^{\prime}\right) & =V(u, v) \\
{\left[\begin{array}{l}
u^{\prime} \\
v^{\prime}
\end{array}\right] } & =\left[\begin{array}{l}
u \\
v
\end{array}\right]+\overline{\bar{U}}\left[\begin{array}{l}
k_{1} \\
k_{2}
\end{array}\right]
\end{aligned}
$$

where

$$
\overline{\bar{U}}=N_{T} d\left[\begin{array}{rr}
0 & \frac{\sqrt{3}}{2} \\
1 & -\frac{1}{2}
\end{array}\right] ; \quad \begin{aligned}
& \overline{u_{1}}=N_{T} d[0,1]^{T} \\
& \overline{u_{2}}=N_{T} d\left[\frac{\sqrt{3}}{2},-\frac{1}{2}\right]^{T}
\end{aligned}
$$

is a sampling matrix in the $(u, v)$ domain. The $\boldsymbol{U}$ matrix is not unique, since all the sampling matrices given by

$$
\begin{aligned}
& \overline{\bar{U}}_{1}=\left[-\overline{u_{1}}, \overline{u_{2}}\right] \\
& \overline{\bar{U}}_{2}=\left[\overline{u_{1}},-\overline{u_{2}}\right] \\
& \overline{\bar{U}}_{3}=\left[-\overline{u_{1}},-\overline{u_{2}}\right]
\end{aligned}
$$

reproduce the same periodic extension in the $(u, v)$ plane. This choice, however, will determine the numbering of the $(u, v)$ and $(\xi, \eta)$ samples to process them properly. The associated periodicity matrix in the $\left(k_{1}, k_{2}\right)$ axes is

$$
\overline{\bar{N}}=\left[\begin{array}{cc}
N_{T} & 0 \\
0 & N_{T}
\end{array}\right] ; \quad \operatorname{det}(\overline{\bar{N}})=N_{T}^{2} \text {. }
$$

If the sampling points in the $(\xi, \eta)$ directing cosines are forced to satisfy the following relation

$$
\overline{\overline{U^{T}}} \cdot \overline{\bar{\Xi}}=\overline{\bar{I}}
$$

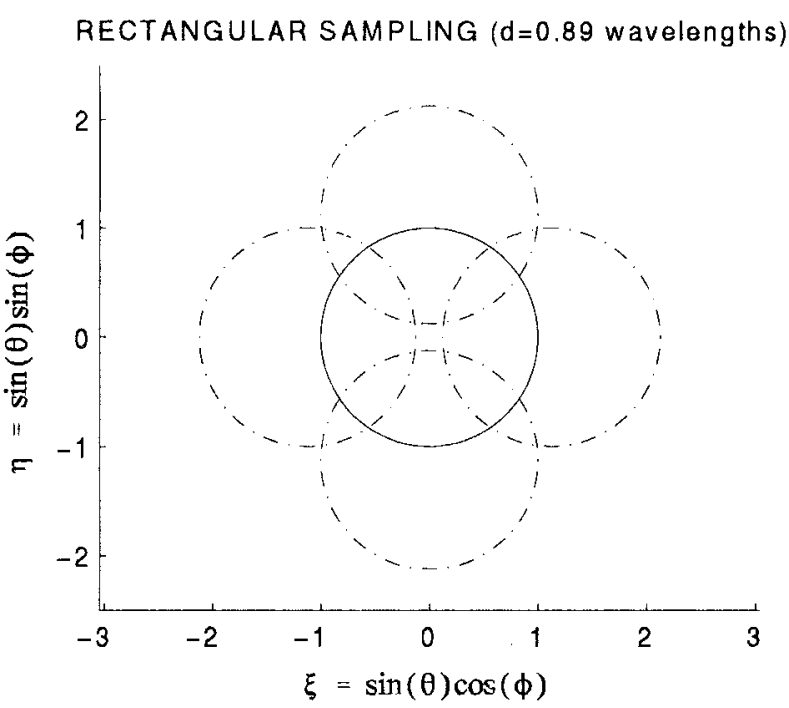

(a)

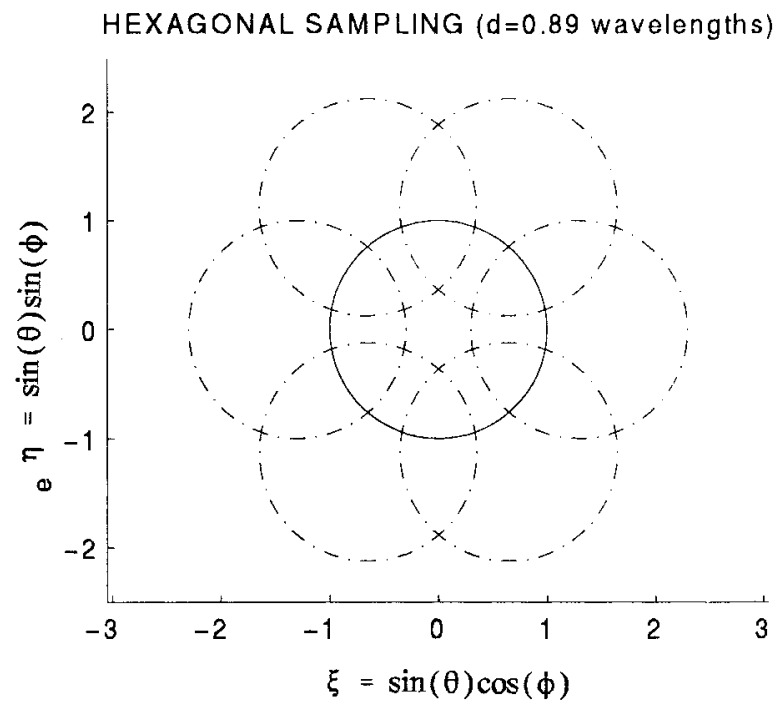

(b)

Fig. 6. Aliasing free regions for (a) $T$-array (rectangular sampling) and (b) $Y$-array (hexagonal sampling) with adjacent antennas spaced $0.89 \lambda$.

then:

$$
\overline{\bar{\Xi}}=\left(\overline{\overline{U^{T}}}\right)^{-1}=\frac{1}{N_{T} d}\left[\begin{array}{cc}
\frac{1}{\sqrt{3}} & \frac{2}{\sqrt{3}} \\
1 & 0
\end{array}\right] \triangleq\left[\overline{\xi_{1}}, \overline{\xi_{2}}\right]
$$

and the Fourier transform kernel becomes separable, even if the $(u, v)$ and $(\xi, \eta)$ sampling points are not chosen over a rectangular grid. $\left[\xi_{1}, \xi_{2}\right]$ form the reciprocal basis of $\left[\boldsymbol{u}_{1}, \boldsymbol{u}_{2}\right]$ in the $(\xi, \eta)$ domain (Figs. 5 and 6). With this concept, the sampled $(u, v)$ and $(\xi, \eta)$ points are given by:

$$
(u, v)=\left(\frac{\sqrt{3}}{2} d k_{1}, \frac{d}{2}\left(-k_{1}+2 k_{2}\right)\right) ; k_{1}, k_{2}: 0 \cdots N_{T}-1
$$




$$
\begin{gathered}
(\xi, \eta)=\left(\frac{1}{\sqrt{3} N_{T} d}\left(n_{1}+2 n_{2}\right), \frac{1}{N_{T} d} n_{1}\right) ; \\
n_{1}, n_{2}: 0 \cdots N_{T}-1 .
\end{gathered}
$$

And the inverse Fourier transform of the hexagonally sampled $V\left(u\left(k_{1}, k_{2}\right), v\left(k_{1}, k_{2}\right)\right)$ is given by

$$
\begin{aligned}
T\left(n_{1}, n_{2}\right) \triangleq & \left.T\left(\xi, n_{1}, n_{2}\right), \eta\left(n_{1}, n_{2}\right)\right) \\
= & \frac{\sqrt{3} d^{2}}{2} \sum_{n_{1}=0}^{N_{T}-1} \sum_{n_{2}=0}^{N_{T}-1} V\left(k_{1}, k_{2}\right) \\
& \cdot \exp \left\{j 2 \pi \left[u\left(k_{1}, k_{2}\right) \xi\left(n_{1}, n_{2}\right)\right.\right. \\
& \left.\left.+v\left(k_{1}, k_{2}\right) \eta\left(n_{1}, n_{2}\right)\right]\right\} \\
= & \frac{\sqrt{3} d^{2}}{2} \sum_{n_{1}=0}^{N_{T}-1} \sum_{n_{2}=0}^{N_{T}-1} V\left(k_{1}, k_{2}\right) \\
& \cdot \exp \left[j \frac{2 \pi}{N_{T}}\left(k_{1} n_{2}+k_{2} n_{1}\right)\right]
\end{aligned}
$$

Expression (23) can be recognized as a standard rectangular FFT with $n_{1}$ and $n_{2}$ interchanged. The factor $\sqrt{3} d^{2} / 2$ is the pixel area in the $(u, v)$ domain. The recovered modified brightness temperature distribution given by (23) is repeated periodically over the $(\xi, \eta)$ domain. The centers of periodic cells can be found by applying the periodicity condition to the argument in the Fourier kernel

$$
\begin{aligned}
\frac{2 \pi}{N_{T}}\left[\bar{u}_{1}^{T}\left(\xi_{n}, \eta_{n}\right)+{\overline{u_{2}}}^{T}\left(\xi_{n}, \eta_{n}\right)\right] \\
\quad=2 \pi\left[(0, d)(\xi, \eta)+\left(\frac{\sqrt{3}}{2} d,-\frac{d}{2}\right)(\xi, \eta)\right] \\
\quad=2 \pi m ; \quad m=0, \pm 1, \pm 2
\end{aligned}
$$

whose solutions closest to the origin are

$$
\begin{gathered}
\left(\xi_{n}, \eta_{n}\right)=\frac{2}{\sqrt{3} d}\left(\cos \left(n \frac{\pi}{3}\right), \sin \left(n \frac{\pi}{3}\right)\right) \\
n=0,1,2, \cdots 5 .
\end{gathered}
$$

If the extension of the modified brightness temperature is the hole unit circle, the $\left(\xi_{n}, \eta_{n}\right)$ points must be at a distance 2 from the origin to avoid aliasing completely, which forces a maximum antenna spacing of $d \leq \lambda / \sqrt{3}$. Compared to rectangular sampling, where the maximum antenna spacing is $d \leq \lambda / 2$ to avoid aliasing, the hardware savings of the $Y$-array are $13.4 \%$. Fig. 6(a) and (b) show the alias free FOV for a $T$ array, rectangular $(u, v)$ sampling, and a $Y$-array, hexagonal $(u, v)$ sampling, whose adjacent antennas are spaced in both cases $d=0.89 \lambda$. It can be observed that the alias free FOV is larger for hexagonal sampling. In the Earth observation situation, the Earth does not occupy the whole unit circle and the antenna spacing condition can be relaxed depending on the required alias free swath (Fig. 7). The $0.89 \lambda$ spacing between antennas in MIRAS is a compromise between array thinning and aliasing free swath, which is about $900 \mathrm{Km}$ [8]. This swath satisfies the three day revisit time necessary to update soil moisture and ocean salinity measurements [1].

In addition, since in the inversion process there is no interpolation neither in the $(u, v)$ domain nor in the $(\xi, \eta)$

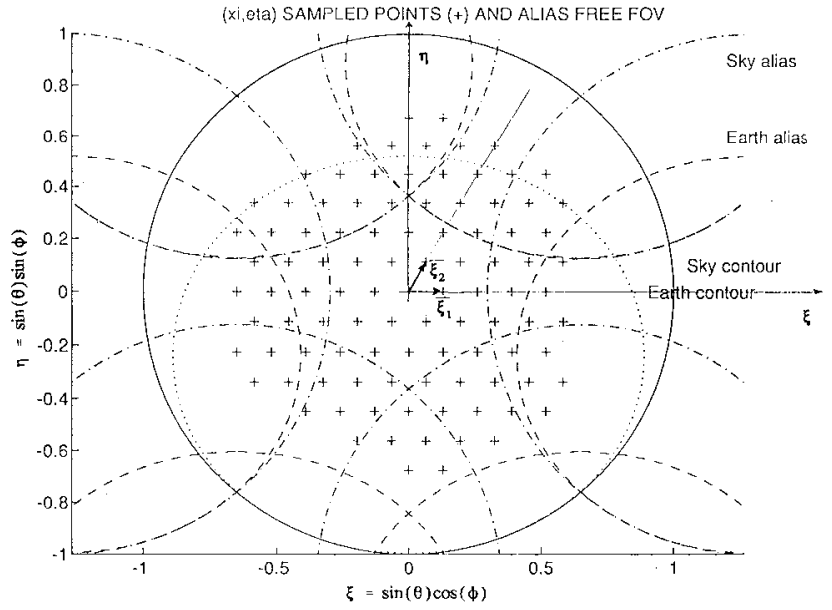

Fig. 7. Sampled points in the directing cosines domain and the reciprocal basis of Fig. 5 basis.

domain, artifacts are not induced in the recovered brightness temperature map and signal to noise ratio is preserved. At this point two important relations between the interferometer's array geometry and the $(u, v)-(\xi, \eta)$ points should be pointed out a) the total number of correlations $\left(N_{T}^{2}\right)$ is equal to the number of samples in the fundamental hexagonal $(u, v)$ and $(\xi, \eta)$ cells $(19)$ and $b)$ the number of redundant correlations between antenna pairs, including the baseline zero, is equal to the number of missing $(u, v)$ visibility samples which will be initially padded with zeros. This technique has been applied to the particular sampling grids given by MIRAS $Y$-array, however it can be used with any other sampling strategy with an appropriate $\Xi$ matrix satisfying (21), (22a) and (22b).

\section{EXAMPLE: APPLICATION TO APERTURE SYNTHESIS RADIOMETERS}

Figs. 8 and 9 show the results of the application of this technique to a case similar to space-borne MIRAS: a Y-shaped interferometer radiometer with 43 antennas per arm spaced 0.89 wavelengths. The platform is at $800 \mathrm{Km}$ and the array is tilted $31.2^{\circ}$ with respect to nadir. The image treated in this example has been taken from MATLAB [(c) The MATH WORKS Inc.] and has been properly modified in order to give realistic brightness temperature values. This image is composed by a ellipsoidal contour representing the Earth-sky border as seen from the satellite in the $(\xi, \eta)$ directing cosines representation (Fig. 8). The sky occupies the zone in between the ellipsoidal contour and the unit circle and its brightness temperature is assumed to be $3 \mathrm{~K}$. The brightness temperature of the sea has been taken $100 \mathrm{~K}$ and that of the coast ranges from $220 \mathrm{~K}$ to $300 \mathrm{~K}$.

The original brightness temperature is shown in Fig. 8(a), from which the set of visibilities have been computed over the $(u, v)$ hexagonal grid given in (22a) according to (1). When computing these values we have assumed that noise due to finite integration time is negligible, consequently any error in the recovered images is due only to the inversion process. Fig. 8(b) shows the brightness temperature map restricted to 
(a)

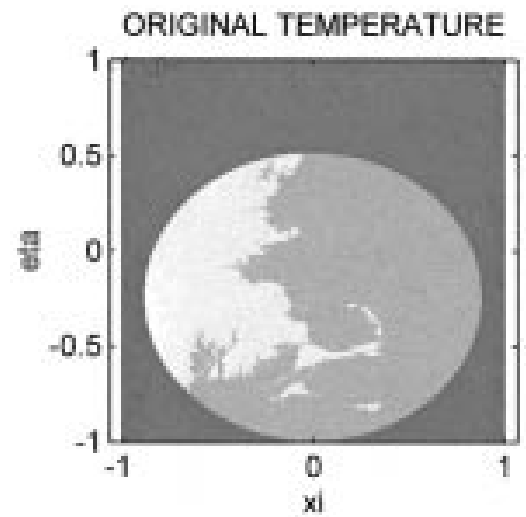

FFT TB (Rectangular Window)

(c)

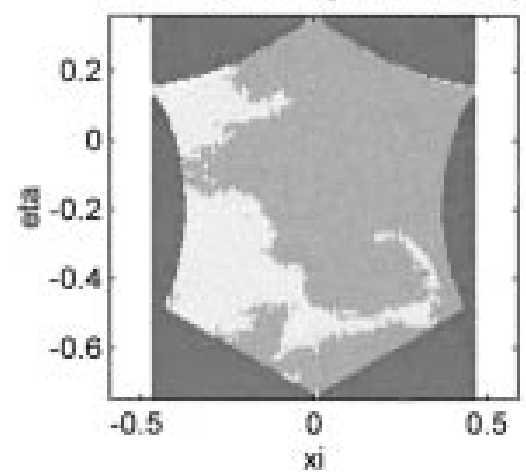

(b)

ORIGINAL TEMPERATURE IN ALIAS FREE FON

(d)

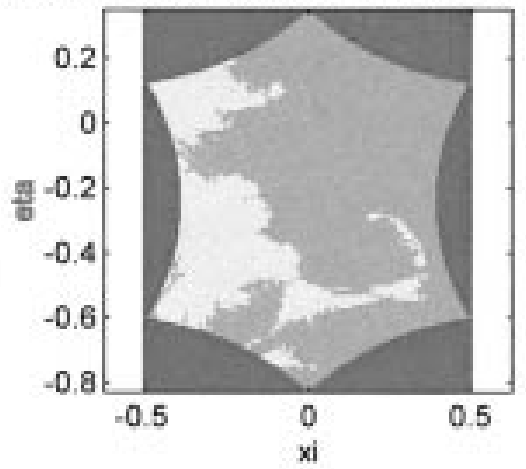

FFT TB (Blackmann. Window)

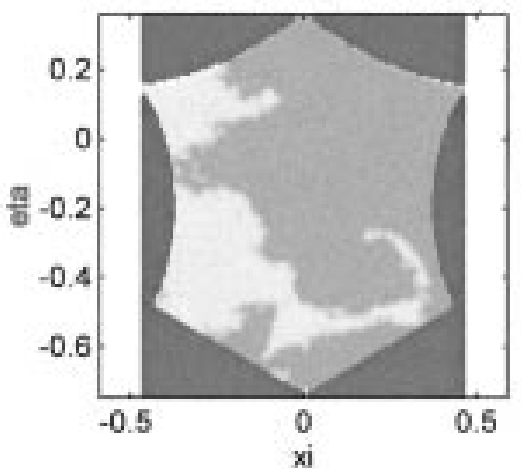

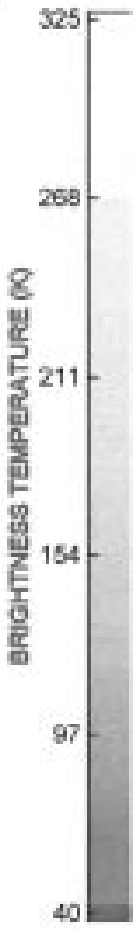

Fig. 8. (a) Hypothetical earth model for hexagonal visibility processing using standard FFT as seen from a $800 \mathrm{Km}$ height, $31.2^{\circ}$ tilted platform. (b) Alias free field of view of Fig. 8(a). Subsampling with 0.89 wavelengths spacing between antennas reduces alias free swath to $900 \mathrm{~km}$. (c) Recovered brightness temperature in the alias free field of view by inverse Fourier transform of hexagonally sampled visibilities with rectangular weighting window. (d) Recovered brightness temperature in the alias free field of view by inverse Fourier transform of hexagonally sampled visibilities with Blackmann weighting window.

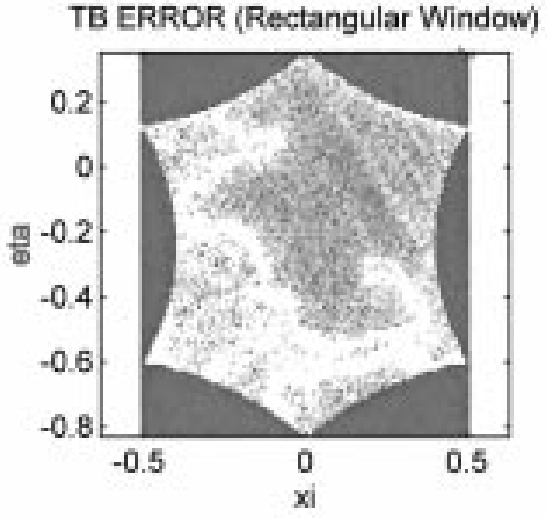

(a)

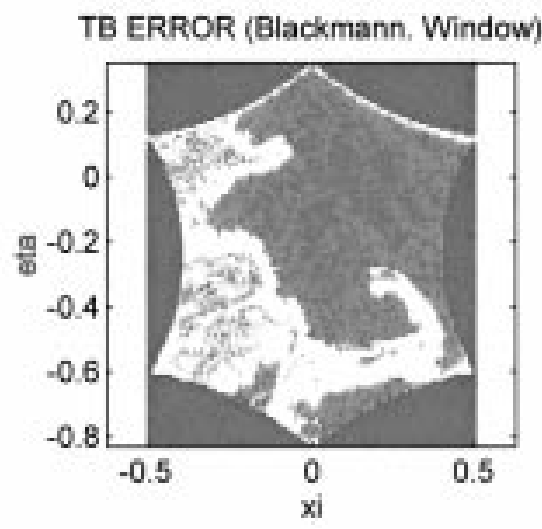

(b)

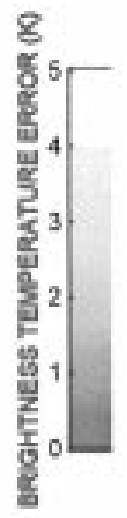

Fig. 9. (a) Error in the recovered brightness temperature with rectangular weighting window [Fig. 8(b) minus (c)]. (b) Error in the recovered brighteness temperature with Blackmann weightning window [Fig. 8(b) minus (d)].

the alias free field of view (FOV). Fig. 8(c) and (d) show the inverse Fourier transform of the visibility samples computed according to (22a) without weighting function (rectangular window) and with a Blackmann window, respectively. Since the spacing between antennas exceeds $1 / \sqrt{3}$ wavelengths, the Nyquist criterion for hexagonal sampling, some aliasing exists (Fig. 7): results are shown cut to the alias free FOV. Note the absence of artifacts, usually of periodic character, that generally appear when interpolations are performed. Note also that the Blackmann windowed image appears more blurred than its rectangular windowed counterpart. Fig. 8(a) and (b) show the discretization and $(u, v)$ finite coverage errors computed as the difference between the recovered brightness temperature maps [Fig. 8(c) and (d)] and the original brightness temperature [Fig. 8(b)]. Note the high errors due to the Gibbs phenomenon at the coast line due the $120 \mathrm{~K}$ step in the brightness temperature. Errors decrease when highly tapered windows are used. The trade-off shown in [12] between 
high spatial resolution, requiring low weighting functions, and high radiometric resolution, requiring highly tapered weighting functions, can be easily detected.

Since the $(u, v)$ coverage is finite, its inverse Fourier transform is not limited and some alias "tails" enter partially in the nominal alias free FOV. In Figs. 8(c) and 9(a) a border pixel has been removed to minimize this effect. However, it is more apparent in Figs. 8(d) and 9(b) because of the wider system's impulse response caused by the Blackmann weighting function. However, aliasing impact in the FOV can be minimized by using some a priori information such as the sky brightness temperature and an average Earth brightness temperature. Aliasing degrades MIRAS performance at swath edges and presents added difficulties in the inversion process since measured visibilities depend also on the brightness temperature from aliased regions. This difficulties can be partially alleviated by restricting the inversion region to a smaller area inside the alias free FOV [7].

\section{CONCLUSIONS}

A proper choice of the interferometer's array configuration allows a substantial reduction of the number of visibility samples and hardware requirements for a determined aliasing level. Y-shaped and triangular-shaped arrays sample the visibility function over a hexagonal grid optimally. Compared to rectangular $(u, v)$ sampling a hardware reduction of $13.4 \%$ is obtained. In addition, Y-shaped arrays provide larger $(u, v)$ coverage than triangular-shaped arrays, thus improving the spatial resolution capabilities of the instrument.

This paper has presented a simple procedure to fully exploit the benefits of the hexagonal sampling grid given by the Y-shaped arrays, as MIRAS: reduces hardware requirements and the number of visibility samples (13.4\%), increases computational speed (25\%) with standard rectangular rowcolumn routines and avoids the drawbacks of hexagonal to rectangular conversion mainly: additional computational load, interpolation induced artifacts and signal to noise degradation. This technique is based on the use of rectangular FFT to process hexagonally sampled signals provided that the $(\xi, \eta)$ pixels are properly chosen over the reciprocal grid of the $(u, v)$ hexagonal grid. However, the proposed technique is not restricted to hexagonal grids and can be used with other sampling strategies, provided that the reciprocal basis is used.

An example of this technique applied to MIRAS has been presented a space-borne Y-shaped interferometer radiometer with 43 antennas per arm. Subsampling problems have been shown: mainly aliasing and radiometric resolution degradation at swath edges.

\section{REFERENCES}

[1] SMOS, "Conclusions and recommendations from SMOS" and "Summary reports of the working groups," Consultative Meeting on Soil Moisture and Ocean Salinity. Measurement Techniques and Radiometer Techniques. ESA WPP-87. ESTEC, Noordwijk, The Netherlands, 20-21 Apr. 1995. pp 6-11
[2] C. S. Ruf, C. T. Swift, A. B. Tanner, D. M. Le Vine, "Interferometric synthetic aperture microwave radiometry for the remote sensing of the earth," IEEE Trans. Geosci. Remote Sensing, vol 26, Sept. 1988.

[3] M. Martín Neira, Y. Menard, J. M. Goutoule, and U. Kraft, "MIRAS, a two-dimensional aperture synthesis radiometer," in Proc. IGARSS 1994, pp $1323-1325$.

[4] A. R. Thompson, J. M. Moran, and G.W. Swenson, Interferometry and Synthesis in Radio Astronomy. New York: Wiley, 1986.

[5] R. M. Mersereau, "The processing of hexagonally sampled twodimensional signals," Proc. IEEE, vol 67, June 1979.

[6] D. E. Dudgeon and R. M. Mersereau, Multidimensional Digital Signal Processing. New York: Prentice-Hall, 1984.

[7] J. Bará, A. Camps, I. Corbella, and F. Torres, "Bidimensional discrete formulation for aperture synthesis radiometers," CNN2 to Work Order No 10 to ESTEC Contract No 9777/92/NL/PB.

[8] MATRA MARCONI SPACE, "MIRAS: Microwave Imaging Radiometer with Aperture Synthesis. Microwave Radiometry Critical Technical Development,” ESA-ESTEC, Final Report., Jan. 1995. ESTEC Contract 9777/92/NL/PB.

[9] E. Gündüzhan, A. Enìs Cetin, and A. Murat Tekalp, "DCT coding of nonrectangularly sampled images," IEEE Signal Processing Lett., vol. 1, Sept. 1994.

[10] R. Bernardini and R. Manduchi, "On the reduction of multidimensional DFT to separable DFT by Smith normal form theorem," European Trans. Telecommun. Related Technol., vol 5, pp. 377-380, May-June 1994.

[11] A. Camps, J. Bara, I. Corbella, and F. Torres, "Visibility inversion algorithms over hexagonal sampling grids," Soil Moisture and Ocean Salinity Measurements and Radiometer Techniques Meeting, ESA-ESTEC. Noordwijk, The Netherlands, Apr. 20-21, 1995.

[12] A. Camps, J. Bara, I. Corbella, and F. Torres, "Radiometric sensitivity computation in aperture synthesis interferometric radiometry," to be published.

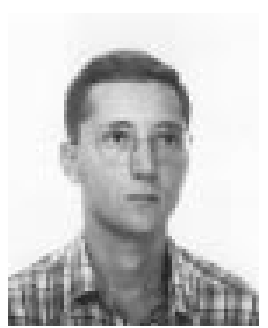

Adriano Camps (S'96) was born in Barcelona, Spain, in 1969. He received his Ingeniero degree in telecommunication engineering from the Polytechnic University of Catalonia (UPC), Barcelona, Spain, in 1992. He is currently pursuing the Doctor Ingeniero degree in the study of interferometric radiometers applied to Earth observation.

In 1991-92 he received an ERASMUS fellowship at the École Nationale Supérieure des Télécommunications de Bretagne (ENST-Br), Brest, France, where he followed the microwave and optical systems branch. In 1992 he joined Eurowaves-Sorep, Rennes, France, as a student-engineer where he worked on power microwave amplifiers. Since 1993 he has been at the Antenna-Microwave-Radar group, Department of Signal Theory and Communications of the UPC as an Assistant Professor.

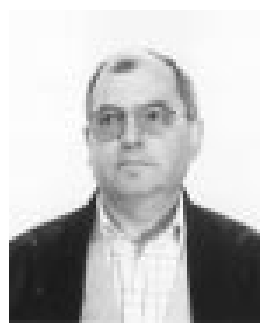

Javier Bará was born on September 30th, 1944. He received the Sc.M. degree in 1968 and the Ph.D. degree in 1972, both in electrical engineering, from Brown University, Providence, R.I.

Since 1972, he has been a Professor at the Polytechnic University of Catalonia (UPC), Barcelona, Spain, where he held several posts of academic responsibility as associate School Dean, Dean and Department Director. He is at present Dean of the College of Telecommunication Engineering 'Baix Llobregat'.

His research interests have been in the field of microwaves (ferrites, integrated circuits, satellite communications, industrial heating and drying processes) and is at present involved in projects in nonguided optical communications in the near infrared and interferometric radiometry for remote sensing of the Earth. 


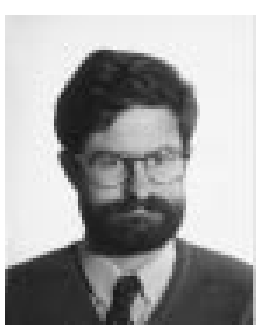

Ignasi Corbella Sanahuja (S'78-M'82) was born in Barcelona, Spain, in 1955. He received the Ingeniero and Doctor Ingeniero degrees in telecommunication engineering, both from the Polytechnic University of Catalonia (UPC), Barcelona, in 1977 and 1983 , respectively.

In 1976 he joined the School of Telecommunication Engineering (ETSET) in Barcelona, Spain, as a Research Assistant in the Microwave Laboratory, where he worked on passive microwave integrated circuit (MIC) design and characterization. In 1979 he joined Thompson CSF at Orsay (France), where he worked in microwave oscillator design and phase noise measurement. He went back to the ETSET and became Assistant Professor in 1982, Associate Professor in 1986, and Professor in 1993. He is currently teaching a full year microwave course. $\mathrm{He}$ is also working in the Department of Signal Theory and Communications of the UPC on several research areas, among which are microwave interferometer radiometry and microwave system design.

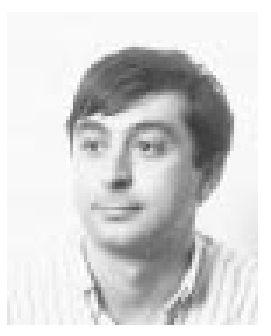

Francesc Torres was born in Ibiza, Spain, in 1962. He received the Ingeniero and Doctor Ingeniero degrees in telecommunication engineering, both from the Polytechnic University of Catalonia (UPC), Barcelona, Spain, in 1988 and 1992, respectively.

From 1988 to 1989 he was Research Assistant in the RF System Division at the European Space Agency, The Netherlands, devoted to microwave device testing and characterization. Since 1989 he has been at the Antenna-Microwave-Radar group of the UPC as an Associate Professor. His main research interests are focused in the design and testing of microwave systems and subsystems. He is currently engaged in research on interferometric radiometers devoted to Earth observation. 